\title{
Guidewires as Embolic Agents? Embolotherapy of Large Splenic Artery Aneurysms
}

\author{
Arafat Muhammed Haris ${ }^{1}$ Jagdeesh Rampal Singh ${ }^{1}$ Chitterusu Raghuram² Rebala Pradeep ${ }^{2}$ \\ D. Nageshwar Reddy ${ }^{3}$
}

1Department of Interventional Radiology, Asian Institute of Gastroenterology, Hyderabad, Telangana India

${ }^{2}$ Department of Gastro-Intestinal Surgery, Asian Institute of Gastroenterology, Hyderabad, Telangana, India

${ }^{3}$ Department of Gastroenterology, Asian Institute of

Gastroenterology, Hyderabad, Telangana, India

\begin{abstract}
Address for correspondence Arafat Muhammed Haris, MD, Department of Interventional Radiology, Asian Institute of Gastroenterology, Hyderabad 500082, Telangana, India (e-mail: arafatharis@gmail.com).
\end{abstract}

J Clin Interv Radiol ISVIR 2021;5:182-185.

\begin{abstract}
Splenic artery aneurysms are among the more frequently diagnosed intra-abdominal aneurysms and are not infrequent in patients with raised portal venous pressure often requiring endovascular or surgical therapy. A 36-year-old female patient with Non-cirrhotic portal fibrosis and portal hypertension was diagnosed with multiple large splenic artery aneurysms for which she was initially operated which resulted in

Keywords

- splenic artery aneurysm

- guidewire

- embolotherapy

- embolic agent substantial blood loss during dissection that required embolotherapy. Initial attempts at coil embolization proved unsuccessful due to the wide aneurysmal neck and flow characteristics. Following which the aneurysmal sac was packed with multiple guidewires to act as a scaffold for further coil embolization. Subsequently, hemostasis was achieved and the patient underwent splenectomy later thereby demonstrating that embolization of large aneurysms can be accomplished with reasonable efficacy using guidewires.
\end{abstract}

\section{Introduction}

Splenic artery aneurysms are among the more frequently diagnosed intra-abdominal aneurysms and are reported to have an incidence of around $8-50 \%$ in patients with raised portal venous pressure, often being the presenting feature of portal hypertension. ${ }^{1,2}$ Management options range from medical management to endovascular and surgical treatment. Here we present a case of Non-cirrhotic portal fibrosis and portal hypertension with multiple large true aneurysms arising along a dilated and tortuous splenic artery that required endovascular coiling using guidewires and standard metallic coils in a two-staged procedure that was followed by surgical aneurysmectomy and splenectomy.

published online April 29, 2021
DOI https://doi.org/

$10.1055 / \mathrm{s}-0041-1728969$ ISSN 2457-0214

\section{Case Report}

A 36-year-old female patient diagnosed with Non-cirrhotic portal fibrosis and portal hypertension was evaluated with a multi-detector computed tomography (MDCT) angiography that demonstrated the presence of a dilated and tortuous splenic artery with multiple large true aneurysms arising from the splenic artery (-Fig. 1). The spleen was massively enlarged with tortuous portal venous collaterals consistent with portal hypertension. The patient was initially taken up for splenectomy. During the surgery, however, the surgeons encountered substantial blood loss during dissection which was dealt by aneurysmorrhaphy and this prompted a plan for a staged procedure; embolization of the splenic artery or
(C) 2021. Indian Society of Vascular and Interventional Radiology. This is an open access article published by Thieme under the terms of the Creative Commons Attribution-NonDerivative-NonCommercial-License, permitting copying and reproduction so long as the original work is given appropriate credit. Contents may not be used for commercial purposes, or adapted, remixed, transformed or built upon. (https://creativecommons.org/licenses/by-nc-nd/4.0/).

Thieme Medical and Scientific Publishers Pvt. Ltd. A-12, 2nd Floor, Sector 2, Noida-201301 UP, India 


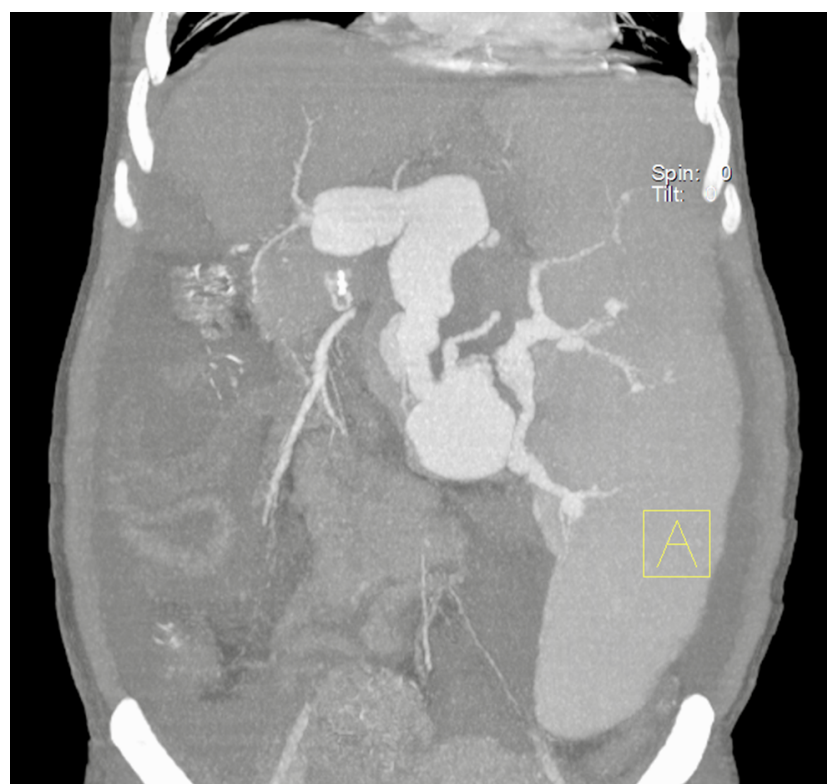

Fig. 1 MDCT angiography in coronal maximum-intensity-projection demonstrating the multiple splenic artery aneurysms.

aneurysms preceding the surgical splenectomy and aneurysmectomy to mitigate profuse intra-operative blood loss. Blood investigations revealed a low hemoglobin $(6.8 \mathrm{~g} / \mathrm{dl})$ for which multiple packed cell units were transfused. Clinical, hematological and coagulation parameters were within normal limits and the patient was taken up for splenic artery embolization.

The right common femoral artery was punctured using a 18G percutaneous puncture needle (Cook Medical, Bloomington, IN) and the celiac artery was accessed using a 5F Simmons catheter (Cook Medical, Bloomington, IN). The subsequent angiogram showed multiple large aneurysms along the course of the splenic artery, the largest aneurysm and the most proximal aneurysm of these measuring $65 \times 49 \mathrm{~mm}$ and $43 \times 35 \mathrm{~mm}$ in size, respectively. The splenic artery at the origin from the celiac trunk measured $19 \mathrm{~mm}$ in diameter with a distance of $36 \mathrm{~mm}$ from the origin to the most proximal aneurysm. Following this, coil embolization was attempted using a $10-\mathrm{mm} \times 14 \mathrm{~cm}$ coil (Nester, Cook Medical, Bloomington, IN) which immediately dislodged into a distal aneurysmal sac due to the wide aneurysmal neck and the intrinsic high flow state that was prevalent. Two other unsuccessful attempts were made to coil embolise the proximal splenic artery with $12-\mathrm{mm} \times$ $14 \mathrm{~cm}$ coils (Nester, Cook Medical, Bloomington, IN) both of which had similarly dislodged into the distal splenic artery immediately on deployment. Another attempt was made to embolize the proximal splenic artery by anchoring a coil into the left gastric artery and deploying into the splenic artery. This again was in vain as the high flow state prevented it from coiling within the splenic artery. The decision to embolize the proximal splenic artery using an Amplatzer vascular plug (AVP) was initially considered however since the intention of endovascular therapy was pre-operative cessation of splenic artery flow to aid surgical ligation, the additional expense that would entail in the likelihood of AVP dislodgement was taken into consideration and hence embolization using AVP was not entertained.

As the usage of coils as embolic agents ended in repeated failures a plan was made to pack the proximal aneurysmal sac with guidewires. This was first attempted using a 0.035 " guidewire (Radifocus, Terumo Corporation, Tokyo, Japan) which was cut according to the length based on the size of the proximal aneurysms and distance from splenic artery to the most proximal aneurysm. Utmost care was taken to ensure the hydrophilic coating was not sheared from the nitinol core so as to prevent the inner core from being exposed. The process was repeated several times, each time cut at a shorter length than the last until the aneurysmal sac was sufficiently packed. After which the angiogram showed persistent filling of the aneurysmal sac, however with reduced flow. Once the proximal aneurysmal sac was packed, multiple coils were nestled into the scaffolding provided by the matrix of guidewires and this was performed using six $12-\mathrm{mm} \times 14 \mathrm{~cm}$, three $10-\mathrm{mm} \times 14 \mathrm{~cm}$ (Nester, Cook Medical, Bloomington, IN) and three $8-\mathrm{mm} \times 5 \mathrm{~cm}$ MReye (Cook Medical, Bloomington, IN) coils which were progressively packed one after the other in to the aneurysmal sac and the proximal splenic artery till a sufficient degree of stasis was achieved meanwhile preserving the right hepatic artery which was seen to arise from the celiac artery (-Figs. 2 and $\mathbf{3}$ ).

The patient was then taken up for surgery two days post-embolization as planned. The splenic artery was clamped at the head of the pancreas and the coils and the guidewires that were placed in the proximal splenic artery were retrieved. As the distal splenic artery was adherent to
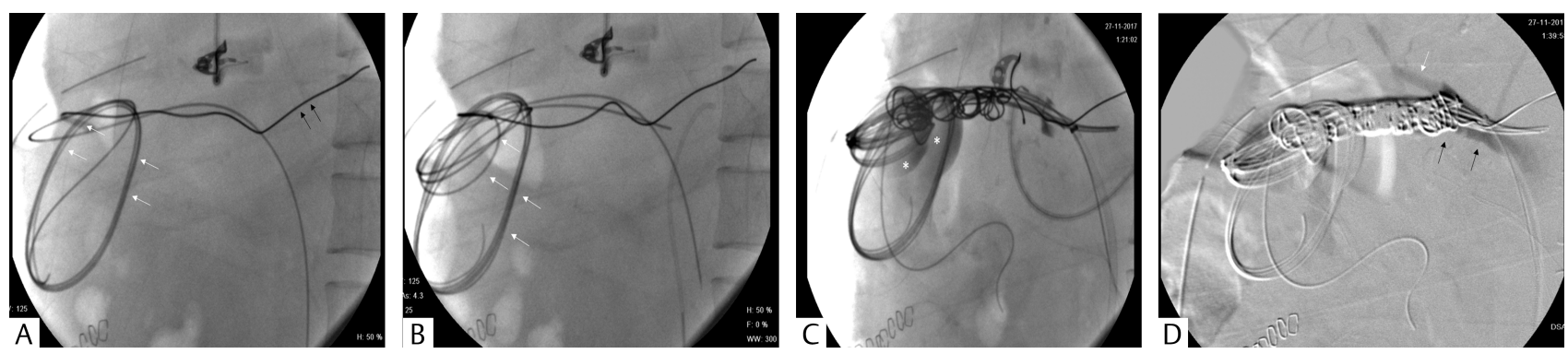

Fig. 2 (A) and (B) Proximal aneurysmal sac with the coiled guidewires within (white arrows). Trailing end of an unsuccessful coiling attempt by anchoring into the left gastric artery (black arrows). Persistent filling of the aneurysmal sac (asterisks) despite multiple guidewires and coils within (C) prompting further deployment of coils until stasis was finally achieved (black arrows) without compromising flow into the right hepatic artery (white arrows) (D). 

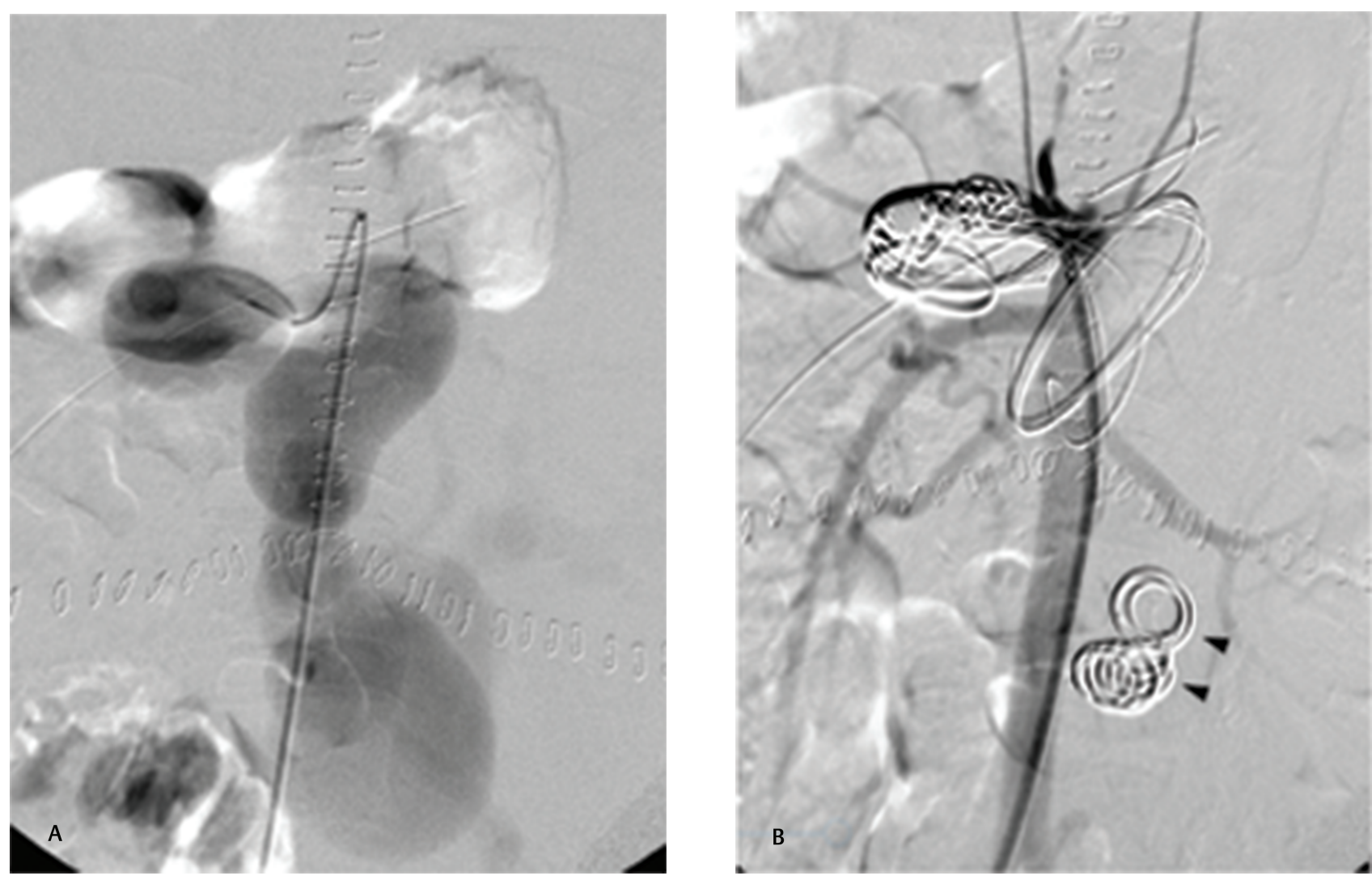

Fig. 3 Pre (A) and post (B) embolization angiograms revealing the multiple aneurysms along the splenic artery and stasis post embolization. Displaced coils from the initial attempts are seen in the distal aneurysmal sac (arrowheads).

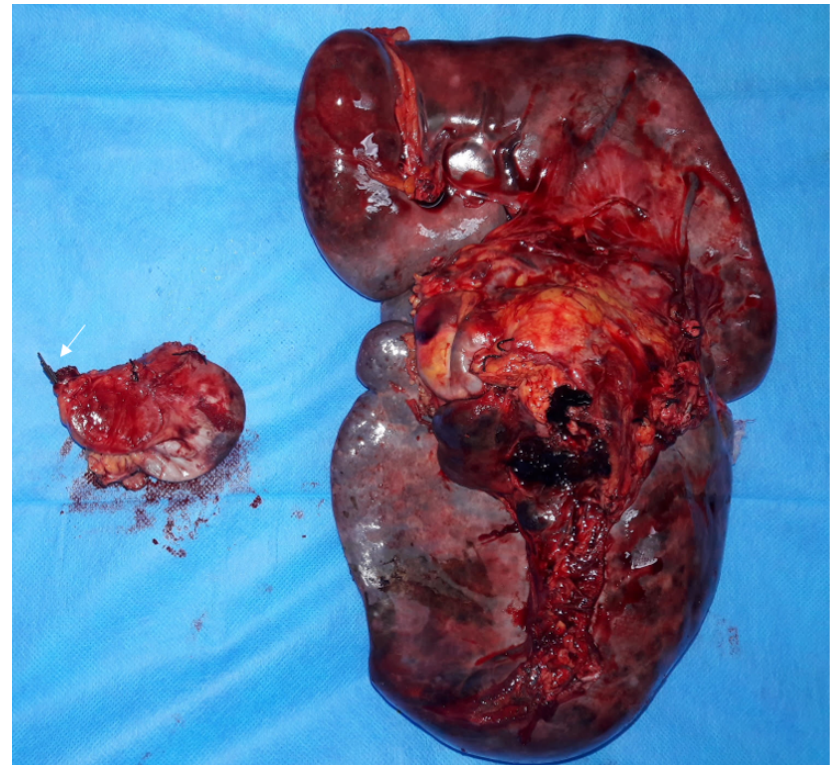

Fig. 4 Surgical splenectomy and aneurysmectomy specimens with a trailing end of one of the guidewires within the proximal aneurysm (white arrow).

the distal body and tail of the pancreas, they were dissected together and resected along with the splenic artery aneurysms and the spleen ( - Fig. 4 ). The post-operative recovery period was uneventful.

\section{Discussion}

Splenic artery aneurysms are among the most common intra-abdominal aneurysms after abdominal aortic and iliac artery aneurysms. Causative factors include multiple pregnancies, arteriosclerosis, trauma, pancreatitis, liver transplantation and portal hypertension. Incidence of splenic artery aneurysms in portal hypertension are reported to range from $8 \%$ to as high as $50 \%{ }^{1}$ The risk of spontaneous aneurysmal rupture and the mortality rate following rupture are as high as $10 \%$ and $40 \%$ respectively and are likely to occur in aneurysms larger than $2 \mathrm{~cm}$ in diameter, pregnancy, pseudoaneurysms and hepatic transplant patients. ${ }^{1,3}$

Endovascular management is considered the first line of management and treatment options include coiling (including detachable coils), glue embolization, stent-graft placement and vessel occlusion by vascular plugs. ${ }^{1,2,4,5}$ Giant aneurysms of the splenic artery are extremely rare with few reported cases managed by surgery. ${ }^{1,6,7}$ The mortality and morbidity associated with surgical treatment of splenic artery aneurysms are $1.3 \%$ and $9 \%$ respectively $^{3}$ and hence in the present case due to the blood loss sustained during the initial surgical attempt the decision was taken to embolize the splenic artery to prevent catastrophic bleeding during surgery. However, the large aneurysmal dilatation and the high flow within the proximal splenic artery precluded packing it with coils. 
The use of guidewires as a matrix to nestle in coils for embolization have been previously described in the embolization of giant pulmonary artery aneurysms and visceral arterio-venous fistulas. ${ }^{89}$ While using the guidewires to pack the aneurysm in our case, initially the decision was taken to use a long enough segment of the guidewire to allow it to coil within the sac and provide a scaffolding for subsequent coiling. There is a risk of arterial wall erosion by the guidewire owing to the stiffness of the inner nitinol core. However, the use of guidewires with Teflon coating in which the inner mandril can be removed will help moderate the risk. In the present case, the benefit of decreased blood loss during the subsequent surgery outweighed this risk since this was a staged procedure and not an exclusive endovascular therapy.

To conclude this case shows that embolization of large aneurysms with unfavorable anatomy and high flow states can be accomplished with reasonable efficacy using guidewires.

\section{Ethical Approval Statement}

All procedures performed in studies involving human participants were in accordance with the ethical standards of the institutional and/or national research committee and with the 1964 Helsinki declaration and its later amendments or comparable ethical standards.

\section{Informed Consent Statement}

Informed consent was obtained from all individual participants included in the study.

\section{Conflict of Interest}

None declared.

\section{References}

1 Lee PC, Rhee RY, Gordon RY, Fung JJ, Webster MW. Management of splenic artery aneurysms: the significance of portal and essential hypertension. J Am Coll Surg 1999;189(5):483-490

2 Akbulut S, Otan E. Management of Giant Splenic Artery Aneurysm: Comprehensive Literature Review. Liu. B, ed. Medicine; 2015;94(27): e1016.

3 Al-Habbal Y, Christophi C, Muralidharan V. Aneurysms of the splenic artery - a review. Surgeon 2010;8(4):223-231

4 Guziński M, Kurcz J, Kukulska M, Neska M, Garcarek J. Embolization of a True Giant Splenic Artery Aneurysm Using NBCA Glue - Case Report and Literature Review. Pol J Radiol 2015;80:155-158

5 Briard RJ, Lee J, Doyle T, Thompson P. Endovascular repair of a portal hypertension-related splenic artery aneurysm using a self-expanding stent-graft. Cardiovasc Intervent Radiol 2009;32(5):1111-1113

6 Yadav S, Sharma P, Singh PK, et al. Giant splenic artery aneurysm: A rare but potentially catastrophic surgical challenge. Int J Surg Case Rep 2012;3(11):533-536

7 Mishra PK, Saluja SS, Sharma AK, Pattnaik P. Management of splenic artery aneurysm associated with extrahepatic portal vein obstruction. Hepatobiliary Pancreat Dis Int 2012;11(3):330-333

8 Trambert JJ, Abubaker SJ, Kanner BJ. Giant mycotic pulmonary artery pseudoaneurysm treated by guide wire and coil embolization. J Vasc Interv Radiol 2014;25(10):1643-1645

9 Kim D, Guthaner DF, Walter JF, Pyle R. Embolization of visceral arteriovenous fistulas with a modified steel wire technique. Am J Roentgenol 1984;142(6):1215-1218 